\title{
Crime au féminin et guerres contemporaines : corrélations
}

Kathryne Fontaine, Université de Toronto

Dans une somme récemment publiée, Coline Cardi et Geneviève Pruvost recensent, selon une approche constructiviste, les grands récits qui ont façonné et qui continuent d'influencer la manière dont la société occidentale conçoit la violence des femmes. La criminalité au féminin, posent-elles, doit se penser à travers les discours historiques, anthropologiques, sociologiques, linguistiques et littéraires, ceux-ci constituant la matière même de la connaissance d'une époque. Plusieurs ouvrages publiés entre autres aux États-Unis et en France au cours des deux dernières décennies poursuivent cet objectif, à l'intérieur cependant d'un cadre heuristique précis : le contexte de la guerre ${ }^{1}$.

La guerre, suggèrent Olivier Odaert et François-Xavier Lavenne, « rompt l'équilibre des sociétés et des vies humaines » et « confronte les individus à l'irrémédiable » (13). Certes, comme l'indique Marc Kravetz,

il n'y a pas que la guerre pour dire la vérité plurielle de notre planète, mais c'est aussi et d'abord peut-être à travers ces conflits sanglants, ces multiples guerres aussi interminables et sauvages que vite oubliées que l'on touche au plus intime le cour de la condition humaine, ce mélange indissociable d'intelligence et de bêtise, de barbarie et de générosité, de mensonge et d'accomplissement de soi... (48)

Phénomène polarisant, la guerre fournit ainsi des perspectives singulières depuis lesquelles envisager, entre autres, la place et les rôles qu'une société, en un temps donné, concède aux femmes, voire lui impose ; en retour, l'angle féminin dans la recherche sur la guerre permet de saisir des enjeux jusqu'ici souvent occultés par l'histoire officielle.

Caron E. Gentry et Laura Sjoberg indiquent toutefois que, dans l'imaginaire populaire nourri en grande partie par les médias, «women who commit acts of violence in defiance of national or international law are not seen as criminals, warriors or terrorists, but as women criminals, women warriors or women terrorists »(8). Le discours qui inscrit leur expérience ne semble jamais pouvoir dissocier leurs actes de leur sexe et les cantonne, en tentant de dresser leur portrait, au modèle de la mère, du monstre ou de la putain. Si c'est là la perception qu'alimente également une certaine doxa dans les domaines de la criminologie féminine et des sciences politiques couverts par Gentry et Sjoberg, il devient pertinent d'explorer des modes d'expression 
plus propices à dépasser ce paradigme. On se demande alors si la littérature de fiction peut constituer un objet pertinent d'étude pour interroger, « quant à sa nature, à l'éthique de son agir, à son identité individuelle et collective » (Odaert et Lavenne 13) une figure aussi problématique que celle de la criminelle de guerre. Forte de sa « dimension narrative » (Cardi et Pruvost 21) et donc capable de recréer des univers et des situations complexes, la fiction peut-elle réellement produire, au sens où l'entend Jean-Jacques Lecercle, de la connaissance (165) ? Peut-elle instiller, à propos des motifs et du statut des femmes qui font le saut, face à la guerre, du côté de la criminalité, des idées auxquelles la doxa n'aurait pas pensé ?

Quelques grandes théories orientent déjà la critique au sujet de la figure de la criminelle en littérature, telle l'idée de l'émancipation de la femme par la violence. Frédérique Chevillot et Colette Trout s'intéressent en ce sens à déterminer si l'action criminelle des femmes « est une forme d'action désespérée et d'auto-défense simplement suicidaire, ou si elle peut représenter [...] une prise de pouvoir au féminin, revendiquant le droit à une violence négativement connotée en passe de devenir constructive » (21-22). Le caractère oxymorique de l'archétype de la femme criminelle est également communément admis, comme l'est l'hypothèse que cette nature contradictoire a contribué à la fascination dont elle a de tout temps fait l'objet. Lecercle affirme en effet : « il y a comme une éternité de la femme criminelle » (167). On s’entend enfin généralement pour dire que la figure est susceptible d'être dépeinte selon des schèmes réducteurs, « fondée sur une simplification par dichotomie qui évacue l'histoire et la politique » (167). Émeline Jouve, Aurélie Guillain et Laurence Talairach-Vielmas remarquent d'ailleurs que, dans le domaine français, «lorsque la représentation de la femme meurtrière dans la littérature et les arts est envisagée, c'est souvent à travers l'étude de figures mythiques relevant d'un passé légendaire plutôt que de l'histoire proche » (15).

Quel est donc le traitement réservé à l'archétype de la criminelle dans les récits fictifs de guerre qui sont contemporains des ouvrages factuels ci-haut nommés ? Il s'avère d'abord que la figure est peu présente. La pièce de théâtre de Wajdi Mouawad (Incendies, 2003) et les romans de Yasmine Char (La main de Dieu, 2008), Ahmadou Kourouma (Allah n'est pas obligé, 2000) et Gil Courtemanche (Un dimanche à la piscine à Kigali, 2004) laissent toutefois croire que lorsqu'il est convié, le personnage de la femme criminelle dans la guerre invite à réenvisager le caractère exceptionnel du crime au féminin et la fixité des rôles typiquement féminins dans la guerre. Non contents de simplement perpétuer dans l'espace fictionnel les paradigmes dominants associés à la 
criminelle, ces œuvres les interrogent à l'aune d'enjeux majeurs auxquels nous confrontent les guerres d'aujourd'hui, tels la responsabilité internationale, le brouillage entre civils et combattants, l'amnistie et le devoir de mémoire. Une analyse des variantes de cet archétype à travers des motifs récurrents qui lui sont associés, tels la marginalité, la sororité et le statut de victime, nous révèle les rapports mutuels qu'entretiennent cette figure et les guerres telles qu'elles se déroulent à notre époque.

\section{La violence genrée révélatrice d'une violence généralisée}

Les personnages féminins criminels font souvent l'objet d'un encadrement narratif qui exacerbe leur caractère marginal, ne serait-ce parfois que sur le plan de la forme. Un dimanche à la piscine à Kigali, par exemple, ne fait que mentionner brièvement Agathe Habyarimana, la femme du président hutu assassiné au début du génocide rwandais. Le roman évoque, dans ce qui ne paraît être que des données contextuelles, la responsabilité de « madame la présidente », d'une part, dans un commerce lucratif d'enfants rwandais avec la Belgique, et, d'autre part, dans l'organisation et la perpétration des massacres des Tutsis. Elle demeure en marge de l'intrigue, constitue plutôt une figure suprême, sorte de marâtre nationale qui vend ses enfants à profit, et l'importance insinuée de son implication dans le conflit est inversement proportionnelle à l'espace qui lui y est aménagé dans le récit. La majorité des détails la concernant se trouve en effet dans une seule note de bas de page :

Le premier avion français repartit de Kigali avec la veuve du président, Agathe Habyarimana, et une trentaine de membres de sa famille, dont quelques-uns des principaux organisateurs du génocide. On remit à la veuve une somme de deux cent mille francs pour subvenir à ses petites dépenses une fois arrivée à Paris. Tous ces assassins vivent librement en France au moment où ces lignes sont écrites. (181)

Ce choix narratif transmet tout de même au lecteur, au-delà de la donnée historique, l'idée du partage de la responsabilité du génocide entre toutes catégories d'humains confondues et de l'insignifiance, devant l'ampleur du désastre, de singulariser la culpabilité d'une femme. Les femmes impliquées dans la corruption et la violence ont souvent été perçues comme marginales, notamment du fait de la rareté des cas que répertorie l'histoire officielle ${ }^{2}$. Le roman de Courtemanche, en n'insistant pas sur la spécificité du caractère féminin de Habyarimana par rapport aux actes commis, fait état de ce que Dominique Rabaté considère la marque de notre 
temps, c'est-à-dire un mal qui est «l'ordinaire [...], la propriété banale et terrible de n'importe qui » («L'abîme », 281).

Cette banalisation de la nature féminine de la criminelle, ou indifférenciation égalitaire (Cardi et Pruvost), se retrouve dans le roman de Kourouma. Allah n'est pas obligé y opère un « effacement partiel des catégories de sexe », sorte de manière de désigner la présence de la fille ou de la femme «sans pour autant théoriser sur cette particularité » du personnage (Cardi et Pruvost 58). Certes, le texte fait ressortir l'incongruité de la figure de l'enfant-soldat de sexe féminin, à mesure que Birahima, le narrateur, en rencontre : «Le plus marrant c'est que, parmi ces enfants-soldats, il y a des filles, oui des vraies filles qui ont le kalach, qui font le faro avec le kalach. Elles ne sont pas nombreuses. C'est les plus cruelles... » (32) ; « Nous avons vu apparaitre un enfant-soldat. Un small-soldier, c'était pas plus haut que le stick d'un officier. Un enfant-soldat en tenue de parachutiste beaucoup trop grande. C'était une fille» (33).

Flora Amabiamina estime que le roman « masculinise et chosifie » (38) les enfants-soldats de sexe féminin en les présentant notamment avec des pronoms masculins ou sémantiquement imprécis. Il m'apparaît plutôt que ces personnages sont, par ces procédés, certes assimilés à leurs homologues masculins, mais dans un grand ensemble qui nie autant l'individualité que confère le genre que celle que procure l'enfance. Le réflexe qu'a le narrateur de considérer comme loufoque le sexe féminin de l'enfant-soldat, contribue davantage à brosser le portrait d'un garçon non éduqué, et donc susceptible d'entretenir les préjugés de sa société, qu'à vraiment ostraciser la jeune fille. À dire vrai, l'homogénéisation des déictiques et le naturel avec lequel Birahima décrit les actes de ces filles soldats (descriptions qui ne sont ni plus ni moins empreintes d'ébahissement que celles portant sur les garçons) contribuent directement à dépeindre le type de conflit que constitue celui du Sierra Leone et du Liberia : une guerre qui non seulement victimise la petite fille, mais corrompt cette figure, symbole de pureté et d'innocence ; qui, en d'autres mots, broie toutes les catégories morales.

Bien sûr, le climat de violence dans lequel évoluent les personnages d'enfants-soldats féminins explique leurs actions. Les descriptions des crimes commis par ces combattantes sont presque systématiquement superposées à des récits qui exposent des circonstances atténuantes. De la jeune Fati qui mitraille deux enfants par accident parce qu'elle est « dans les vapeurs » (57) de haschich, à Sarah qui tente de tuer son compagnon parce qu'elle est « devenue complètement dingue » à force de «fumer du hasch » et de «croquer de l'herbe » (54), Birahima les érige en 
véritables victimes du conflit armé. Sarah a ainsi droit, lors de sa mort, à sa propre « oraison funèbre », honneur que le narrateur ne réserve qu'à quelques-uns de ses amis, tous genres confondus :

D'après mon Larousse, l'oraison funèbre c'est le discours en l'honneur d'un personnage célèbre décédé. L'enfant-soldat est le personnage le plus célèbre de cette fin du vingtième siècle. Quand un soldat-enfant meurt, on doit donc dire son oraison funèbre, c'est-à-dire comment il a pu dans ce grand et foutu monde devenir un enfant-soldat. Je le fais quand je le veux, je ne suis pas obligé. Je le fais pour Sarah parce que cela me plaît, j'en ai le temps et c'est marrant. (55)

Orpheline de mère dès l'âge de cinq ans, abandonnée par son père, exploitée par sa tutrice, battue, violée, contrainte à mendier puis à se prostituer, Sarah en vient à « entrer dans les soldats-enfants pour ne pas crever de faim » (56). La violence de ces filles ne réfère ainsi aucunement à ce que Chevillot et Trout appellent une prise de pouvoir au féminin. Leurs crimes ne sont ni héroïques, ni subversifs, ni émancipateurs : ils perpétuent aveuglement les mouvements d'une sorte de « machine devenue folle et qui va au bout de sa logique excessive » (Rabaté, «Logique », 106) de violence et de destruction. On ne pourrait brosser de tableau plus désolant, ce à quoi Kourouma s'emploie du début à la fin de son récit. En dépeignant à quel point la guerre tribale profane la pureté et l'innocence d'une figure que l'on n'associe traditionnellement pas au crime et à l'agressivité - l'enfant, et de surcroît la petite fille -, l'œuvre traduit le souci de représenter la gravité des conséquences des conflits armés sur les valeurs humaines.

Les rôles traditionnels attribués au genre féminin servent en ce sens, dans Allah n'est pas obligé, d'étalon de mesure de l'instabilité de la société en guerre. Les femmes adultes y sont également introduites avec des précisions concernant leur sexe, qui font ressortir leur caractère distinct : «Le général Baclay était une femme. (On devrait dire générale au féminin. Mais, d'après mon Larousse, 'générale' est réservé à la femme d'un général et jamais au général lui-même.) » (64). La présence inusitée de telles figures devient le vecteur d'une réflexion sur un contexte social dont les enjeux dépassent ceux du genre sexuel. C'est par exemple le cas de sœur Gabrielle Aminata, « une garce de matrone à la mitraillette rapide » (115) et chef d'une brigade de filles soldats dont elle protège la virginité, n'hésitant pas à mitrailler celles « qui se laissaient aller » et « ceux qui violaient les filles » (117). C'est ainsi après avoir tué le responsable du viol d'une enfant que sœur Aminata engage une bataille contre les « chasseurs », où elle trouve la mort :

Bien que femme, elle avait tenu un siège de deux semaines contre deux régiments de chasseurs ; elle avait tué dans des sorties nocturnes neuf chasseurs et elle était 
morte sur une automitrailleuse. Elle méritait amplement les funérailles des héros, des maîtres chasseurs. Et cela quel qu'ait pu être son sexe. C'est pourquoi sœur Aminata a eu les funérailles de maître chasseur, de grand maître chasseur. (118-19)

Par sa mission de protectrice, une femme telle que sœur Aminata aura pu être qualifiée de «véritabl[e] humanist[e]» (Amabiamina 44). Une analyse de l'ambiguïté du personnage laisse toutefois penser que le roman interdit toute classification de ses personnages selon des catégories conventionnelles, et crée plutôt son propre système axiologique, logique interne d'ailleurs validée par un des leitmotivs du narrateur : «c'est la guerre tribale qui veut ça » (118).

Quoique Birahima décrive sœur Gabrielle Aminata comme « une sorte de grande sœur et de mère » (117) pour les membres de la brigade, son récit candide laisse paraître que la frénésie du personnage prévaut sur le bien-être de cette communauté de filles et de femmes. «Cette mission de protection de la virginité avec le kalach était accomplie avec beaucoup de rigueur et sans le soupçon d'une petite pitié » (117), raconte Birahima, dépeignant une femme ayant improvisé sa fonction au gré des troubles politiques du pays, et qui profite de cette brèche dans le tissu social pour intervenir à sa manière dans le conflit. Comme le constatent Cardi et Pruvost, cependant,

brouiller les stéréotypes ne suffit pas à transformer le monde et à s'émanciper des rôles normatifs et des pouvoirs dominants. L'inclusion dans la violence politique se fait d'ailleurs souvent en reprenant les codes de la masculinité sans que cela s'accompagne d'une remise en cause des valeurs traditionnelles et des hiérarchies de genre, qui peuvent même en être paradoxalement confortées puisque le masculin demeure l'aune de mesure. (85)

Et de fait, Aminata a établi son camp près de «la ville stratégique de Mile-Thirty-Eight », dans « la région diamantaire et aurifère », celles des «zones de production de café, de cacao, de palmiers à huile » (104); elle est présentée comme «tiers musulmane, tiers catholique et tiers fétichiste »; elle a « le grade de colonel parce qu'elle avait une grande expérience des jeunes filles pour avoir excisé près de mille filles pendant vingt ans » (116). Derrière l'apparence de protectrice des femmes que décrit le narrateur du roman et à laquelle la réduit parfois la critique, se cache un individu qui use des mêmes moyens oppressifs et arbitraires que ceux qu'emploient les détenteurs illégitimes de pouvoirs mercantiles, religieux et militaires au cours de la guerre civile en Sierra Leone.

Kourouma a ainsi recours à la figure équivoque d'une criminelle de guerre dont la noblesse de la mission est indéniablement assombrie par les moyens qu'elle emploie. Le récit évoque de cette manière plusieurs phénomènes propres aux pays ouest-africains tels le Libéria et la Sierra 
Leone et aux conflits armés qu'ils ont connus. On y aborde certes l'assujettissement des jeunes filles aux pratiques traditionnelles comme l'excision et le mariage conditionnel à la virginité, mais surtout un ordre de valeurs inusité dans lequel des individus, d'une part, commettent un viol collectif puis tuent celle qui cherche à venger leur victime en les attaquant, mais qui, d'autre part, honorent en grandes pompes la mort de celle même qu'ils ont tuée, ses qualités de « maître chasseuse » lui valant, au-delà de l'affront qu'elle leur a fait, les égards d'une « héroïne de guerre » (119). Les gestes et le destin ambivalents de personnages comme Aminata participent de la contemporanéité de ce récit de guerre : cette protectrice qui menace, cette sœur qui assassine, cette ennemie que l'on révère, contribue à « la représentation d'une absence radicale de tout dehors à l'état de guerre » (Rabaté, «Logiques », 107), d'un conflit qui contamine tout, les figures traditionnelles et les repères moraux, et qui ne laisse pas entrevoir de sortie. Dans l'univers de Allah n'est pas obligé, en fin de compte, les actions des criminelles font bel et bien état d'un monde où la violence est désormais le principe supérieur qui façonne les mœurs.

\section{Le crime ou l'origine d'un moi autre}

L'empire de la barbarie de la guerre sur les relations humaines est également à l'œuvre dans le récit de Char. La main de Dieu raconte comment une jeune fille de quinze ans acquiert son «visage de tueuse » pendant la guerre du Liban, au gré des drames familiaux et d'une idylle amoureuse avec un franc-tireur qui se fait passer pour un correspondant étranger. Tueuse, elle le devient bel et bien, meurtrie dans l'amour qu'elle portait à son amant, cet ange gardien qui se révèle au final être un ange de la mort, et qu'elle assassine de sang-froid lorsqu'elle découvre la vérité. La narratrice de ce roman d'apprentissage relate en une série de retours en arrière, d'une part, son enfance et sa posture résistante devant la peur des balles de francs-tireurs, les convenances, la morosité ambiante ; d'autre part, son crime, puis sa capitulation face à ce qui lui paraît une situation sans issue.

De mère française, et fréquentant de surcroit le lycée français au lieu de l'école locale musulmane, la jeune Libanaise, jamais nommée, refuse l'apparence traditionnelle des filles de son pays, enfilant des bottes militaires et un pantalon «qui nie la robe » (15) imposée par la famille. Coiffée « à la Jean Seberg », elle fait scandale, alors que ses compagnes de classe « ont des nattes de princesses orientales » (16). La langue même résiste à sa présence, elle qui traverse chaque jour la ligne de démarcation, cette fille dans « ce no man's land [...]. Cette terre d'aucun homme, sauf 
moi » (13; j’ajoute les italiques). «Je ne suis pas une fille, je suis un soldat, avec mon âme, avec mon sang, je libérerai ma patrie » (43), affirme la protagoniste, résolue à s’embrigader malgré son sexe. «Cela m'enchante, déjà à cet âge, d'être en dehors »(15), admet-elle. De fait, cette marginalité forme le nœud de l'intrigue : constatée dès l'enfance, puis farouchement assumée, elle devient, après le crime, ce de quoi la jeune fille souhaite s'éloigner. Le roman se clôt ainsi : «Elle allait tranquillement rejoindre son père et lui obéir. Elle voulait apprendre à marcher comme les autres. Comment faisaient-ils ? Un pas après l'autre, marcher doucement dans la quiétude des jours heureux » (97).

Comment s'opère donc un tel revirement ? A-t-on affaire, dans ce roman de Char, à ce que Cardi et Pruvost appellent la violence féminine d'exception, celle qui survient comme dans une parenthèse, une violence de l'ordre du carnavalesque, qui bouleverse toutes les catégories sociales, mais pour un temps seulement, faisant de la transgression représentée un «non-événement» (Cardi et Pruvost 43-44) ? Son crime est ainsi raconté :

Elle avait eu un hoquet de surprise [...]. À proximité de la fenêtre, il y avait un fusil à lunette : le fusil du franc-tireur. Il n'était pas correspondant de guerre. Il était celui qui avait menti depuis le début [...]. Elle pleurait, elle riait, elle insultait le destin. La jeune fille avait posé son œil contre la lunette du fusil. Il fallait qu'il paie pour tout [...]. Soudain, l'amant s'était trouvé dans le viseur. [...] Sa main n'avait pas tremblé lorsque le coup était parti. Elle n'avait pas vérifié dans l'œil de la lunette si elle l'avait tué, c'était inutile. En sortant, elle avait posé son pistolet en argent sur la table. Elle avait aussi retiré les belles sandalettes. Elle n'aurait plus besoin de courir. (97)

Le crime commis dans les toutes dernières lignes du roman est bel et bien un geste vengeur commis dans le vif de l'émotion, un geste qui range toutefois la protagoniste dans la catégorie des individus qu'elle-même a jadis conçu comme irresponsables et impudents. «Les adultes ne nous expliquaient pas la guerre » (36), raconte en effet au préalable la jeune fille, qui se forme alors sa propre vision de la violence qui l'entoure : «Quand je pense au Liban, je vois un homme qui se soulage. L'individu a de multiples nationalités, il a le visage de l'Orient et de l'Occident, il n'est pas chez lui et, de ce fait, il se comporte en voyou. Il décharge sa haine, il remonte sa braguette et il s'en va » (37). C'est exactement ce qui survient à la fin du récit de la narratrice, lorsque se termine, en quelque sorte, son apprentissage parallèle de la brutalité de la guerre et de l'amour, lorsque la tromperie de son amant lui fait comprendre qu'elle a été « si naïve » (97) : cette fille à la double nationalité décharge le fusil à lunette sur l'homme, se soulageant de sa haine. Mettant un terme à cette relation charnelle interdite, la fille remonte métaphoriquement sa braguette et 
rejoint l'ordre social exigé par sa famille et son pays. L'exil en Suisse évoqué au cours du roman est replacé dans le fil chronologique; elle s'en va.

Elle finit ainsi par incarner en tous points l'image, initialement abhorrée, qu'elle se fait de son pays. Malgré sa nature féminine, son jeune âge et sa nationalité mixte, malgré, donc, ces caractéristiques qui auraient dû, selon une conception traditionnelle des rôles, la maintenir hors de la guerre, elle s'y implique pleinement. Avec cette figure, le roman broie certainement «l'opposition entre un pôle masculin actif, responsable et dominant et un pôle féminin passif, irresponsable et dominé [et] invers[e] symboliquement la norme de l'opposition entre l'autonomie de la force de l'âge et les dépendances multiples de l'enfance » (Jouve et al 21). Encore plus, cependant, il sème l'équivoque quant à la droiture morale de la narratrice, cette héroïne qui tue sans affronter, qui combat sans triompher. Car, si le crime de la jeune fille coïncide avec la naissance de sa lucidité, il scelle du même coup le sort de sa liberté. L'idée d'une quelconque prise de pouvoir est mitigée. Cet adjectif de «tueuse » devient-il réellement, comme elle l'affirme, son « laissez-passer pour une vie meilleure » (9) si ce passage se fait au prix de la soumission à la volonté du père et aux codes sociaux associés à son sexe ? Elle cesse de résister, consciente, peutêtre, d'être désormais aussi coupable que n'importe quel voyou qui, dans la représentation qu'elle s'en était préalablement faite, est responsable de la guerre. Gommer sa marginalité, assurer, du moins en apparence, le « retour à l'ordre social » (Cardi et Pruvost 43), paraît l'unique manière pour la narratrice, « fatiguée de courir » (97), de continuer à vivre.

C'est là, semble-t-il, la seule issue possible pour une jeune fille dans les circonstances que dépeint le roman. Celui-ci rapporte en effet l'histoire de Layal, une compagne de classe de la protagoniste, qui « avait couché. Tout le monde le savait» (17), et qui se voit ainsi rejetée par la famille de son fiancé. Layal et la narratrice, à l'opposé l'une de l'autre, sont attirées l'une par l'autre :

Layal, beaucoup plus belle que moi, la farouche avec cet accoutrement bizarre et cet air bravache. Elle, elle est sans véritable résistance, une fille aimante, prête à se laisser coudre pour racheter sa faute et à accepter un mariage arrangé. C'est possible de lui demander ce genre de choses, de renoncer à ses aspirations. Au contraire de moi, Layal pleine de tout, jamais affamée, tranquille. (55)

La jeune fille voit dans Layal, dont la docilité et le caractère résigné l'impressionnent, une confidente, la seule à qui elle peut parler de son amant et de la perte de sa virginité. Et Layal révèle en retour son estime pour la narratrice au cours d'un moment où elle l'embrasse : «Elle a dit : 
excuse-moi, j'avais besoin de ta force » (56). Layal et la narratrice forment à elles seules une sororité. Elles se mirent l'une dans l'autre, cultivent l'un des rares liens affectifs exempts de violence dans le roman, cet univers où « les rapports d'amour sont semblables à la guerre : partout s'introduire et saccager » (27). Leur baiser témoigne d'une solidarité féminine « dans un pays où les femmes sont piégées » (56). Leur brève amitié est l'occasion pour le récit de montrer l'impasse dans lequel se retrouvent les jeunes filles libanaises, qu'elles « capitule[nt]» (55) et rachètent immédiatement l'honneur de leur famille au prix d'une virginité reconstruite, ou qu'elles résistent plus longtemps et fassent un détour par le crime avant de se ranger comme les autres.

Char exploite donc pleinement les lieux communs relatifs au genre pour situer ses personnages par rapport au contexte, mais surtout pour mettre en évidence le caractère excessivement déroutant de la guerre civile. Happant les individus sur son passage, la guerre les rejette dans le monde, désormais définis par leur expérience, sans que rien toutefois n’y paraisse, sans que l'équilibre des sociétés, dont parlent Odaert et Lavenne, n’en soit véritablement atteint. Le Bildung de la narratrice de La main de Dieu cesse de fait tout de suite après le crime ; lorsque la guerre, en d'autres mots, en a fini avec elle, « Je me souviens que les souvenirs se sont arrêtés lorsque j'ai quitté mon pays. Après, il y a de pâles émotions, rien qui vaille la peine d'être évoqué » (65). L'exil, l'histoire officielle plus friande des actions d'éclat que des états d'âme, la persistance des stéréotypes qui travestissent les faits, contribuent tous à cette sorte d'état de stagnation qui suit l'action. Or cette femme, comme tant d'autres, dont l'histoire personnelle a été interrompue, est restée figée dans la fatalité d'un instant déterminant, fait pourtant matière de ses souvenirs et livre un récit empreint de lucidité.

«[V]ous êtes une tueuse » (9), lui chuchote à l'oreille un étranger, anecdote qui ouvre le roman et enclenche le processus de remémoration. L'organisation narrative propre au récit analeptique permet à la narratrice, comme elle l'exprime, «d'assembler les morceaux de [s]a vie d'avant » (11) et de dénouer l'écheveau de ses souvenirs malheureux et coupables. Seule l'écriture, en d'autres mots, lui procure les moyens de déconstruire l'identité de tueuse qui la définit depuis ce moment paroxystique du meurtre de l'amant. Elle comprend que son «visage de tueuse » est moins l'aboutissant de son crime que la somme de l'abandon de sa mère, du mépris de sa famille, du sentiment d'impuissance devant les massacres perpétrés si près de chez elle, de la honte de la féminité instillée aux jeunes filles dans son pays et de l'ignorance dans laquelle celles-ci sont maintenues : «Il n'est pas apparu soudainement comme je l'ai cru. Ce ne sont pas les bombes qui 
ont creusé les joues. Le visage était en préparation depuis longtemps, la guerre l'a révélé. Maintenant je vois clairement » (12). Le crime opère donc une cassure au cœur de l'expérience de la jeune fille chez Char : «Il y a eu un avant et il y a eu un après », raconte-t-elle (95).

Mouawad exploite également le motif de l'événement décisif qui anéantit un individu mais qui, par les ressorts de la narration, fonde un récit plus perspicace à partir duquel il peut mieux se comprendre. Inspirée de l'histoire de Souha Béchara, Incendies place le crime de guerre au centre d'une réflexion sur la responsabilité et la mémoire. Le personnage de Nawal Marwan y rappelle de réelles figures du mouvement national palestinien. Pour la plupart éduquées, voire occidentalisées, ces femmes souvent jeunes sont mues par des motivations politiques et prêtes à se sacrifier pour la cause. Le discours médiatique qui les entoure demeure toutefois, remarque Sonia Dayan-Herzbrun, superficiel et stéréotypé. Quand elles ne deviennent pas des icônes, elles sont immanquablement issues de la misère sociale ou alors instrumentalisées par des hommes (142). Le portrait que dresse Mouawad de Nawal transcende cependant les clichés. Il relève plutôt du tableau exhaustif que du schéma, même si le personnage féminin y présente certains aspects d'une figure mythique : «On ne prononçait pas son nom. C'était la femme qui chante. Le numéro 72. Cellule no 7. Celle qui a assassiné le chef des milices. Deux balles. Le pays a tremblé. Ils l'ont mise à Kfar Rayat. Tous ses amis ont été attrapés et tués. L'une d'elles s'est rendue jusqu'au café où se tenaient les miliciens et s'est fait exploser » (93-94). La pièce entre toutefois dans les détails de son intimité et refuse de l'héroïser. Elle est d'entrée de jeu présentée comme un personnage énigmatique, incompris : ayant été silencieuse pendant les cinq dernières années de sa vie, elle laisse à ses enfants un testament aussi insolite que mystérieux, déclencheur de la quête qu'entameront ceux-ci pour découvrir la vérité au sujet de leur mère.

L'assassinat que vient à commettre Nawal est d'abord ancré dans sa condition de victime. À la recherche d'un fils qu'on lui a enlevé à la naissance car conçu hors mariage, Nawal, accompagnée de Sawda, parcourt un pays innommé mis à feu et à sang par la guerre civile. Suite à des massacres survenus dans des camps de réfugiés - dont la description rappelle les carnages réellement perpétrés à Sabra et Chatila au Liban en 1982 - les deux femmes se sentent placées devant une sorte d'impératif moral d'agir :

Alors on fait quoi ? On fait quoi ? On reste les bras croisés ! On attend ? On comprend? On comprend quoi ? On se dit que tout ça, ce sont des histoires entre des abrutis et que ça ne nous concerne pas! Qu'on reste dans nos livres et notre alphabet à trouver ça «tellement » joli, trouver ça «tellement» beau, trouver ça 
« tellement » extraordinaire et «tellement» intéressant ! « Joli. Beau. Intéressant. Extraordinaire »sont des crachats au visage des victimes. Des mots ! À quoi ça sert, les mots, dis-moi, si aujourd'hui je ne sais pas ce que je dois faire! On fait quoi, Nawal ? (86-87)

Si Nawal refuse de s'en remettre, comme Sawda, à la loi du talion, sa haine est tout de même « grande, très grande envers ces hommes » (89). « [M]oi la première », jure-t-elle, « je prendrais les grenades [...] et tout ce qui peut faire le plus de mal, je les enroulerais autour de moi, je les avalerais, et j'irais tout droit au milieu des hommes imbéciles et je me ferais exploser avec une joie que tu ne peux pas même soupçonner » (88). Le récit met en opposition les hommes, agents actifs de la guerre, puis les femmes, qui y réagissent ou qui résistent. «[O]n est démunies », explique Nawal. « [O]n n'aime pas la guerre, et on est obligés de la faire » (87). Leur recours à la violence ne s'inscrit toutefois pas dans le cadre de revendications féministes explicites : il est plutôt le résultat d'« une sorte de 'socialisation' de l'homicide comme moyen de subsistance » (Cardi et Pruvost 14). Cela paraît évident lorsque Sawda abat un milicien qui prend en joue les deux femmes, et que Nawal, dans une réplique antithétique qui affirme leur posture défensive, justifie le geste de son amie : «Tu ne l'as pas tué, tu nous as gardées en vie » (81).

De son côté, la décision de Nawal d'assassiner le chef des milices constitue le moyen pour elle de « sortir de la haine » (89) et de permettre à d'autres de connaître l'amour qu'elle a jadis vécu avec le père de son enfant: «Pourquoi on va faire tout ça ? Pour se venger ? Non. Parce qu'on veut encore aimer avec passion. [...] ceux qui ont déjà aimé avec passion doivent mourir avant ceux qui n'ont pas encore aimé. [...] Moi, l'amour que j'avais à vivre, je l'ai vécu [...] Alors il ne me reste que ma mort et je la choisis et elle sera entière » (91). Une promesse de s'éduquer faite à Nazira, sa grand-mère, est à l'origine de cette détermination à ne pas simplement assouvir sa vengeance mais à opter pour un acte réfléchi, délibéré, susceptible d'avoir une plus grande portée.

NAZIRA : Nous, notre famille, les femmes de notre famille, sommes engluées dans la colère depuis si longtemps : j'étais en colère contre ma mère et ta mère est en colère contre moi tout comme toi, tu es en colère contre ta mère. Toi aussi tu laisseras à ta fille la colère en héritage. Il faut casser le fil. Alors apprends. Puis va-t'en. (42)

La condamnation à mort que signifie pour Nawal cet assassinat est le passage obligé vers la paix pour toute une lignée de femme.

Nawal, qui ne meurt cependant pas après avoir commis son crime, subit en guise de représailles l'emprisonnement, la torture et le viol, perpétré de surcroît, comble de l'avilissement, 
par son propre fils perdu à la naissance, et devenu milicien. Comme l'avait prédit Nazira, elle a transmis la colère à ses enfants. Or la promesse tenue finit par avoir des conséquences à long terme. Ce qui permettra à Nawal de s'affranchir de la violence sera le legs de sa mémoire, de son témoignage :

Nous n'aimions pas la guerre ni la violence, nous avons fait la guerre et avons été violents. À présent, il nous reste encore notre possible dignité. Nous avons échoué en tout, nous pourrions peut-être sauver encore cela : la dignité. Vous parler comme je vous parle témoigne de ma promesse tenue envers une femme qui un jour me fit comprendre l'importance de s'arracher à la misère : "Apprends à lire, à parler, à écrire, à compter, apprends à penser [...]. Mon témoignage est le fruit de cet effort. Me taire sur votre compte serait être complice de vos crimes ». (104)

Le crime commis par cette femme prise dans la guerre perpétue donc le cycle des violences et paraît en cela paradoxal de la part de celle qui prêche la réflexion au-delà de la vengeance. Il s'avère toutefois ultimement comme le sacrifice - l'incendie - nécessaire pour que naisse des cendres du fil de la colère et de l'ignorance un nouvel ordre social basé sur le lien affectif, une fille-iation qui « remonte au jour où une jeune fille revint à son village natal pour y graver le nom de sa grand-mère Nazira sur sa tombe » (132).

$* * *$

Les œuvres étudiées ici, si elles ne résolvent pas les antinomies inhérentes au personnage de la criminelle, dissèquent une figure déconcertante du point de vue de l'ordre social, et contribuent ainsi à éroder la vision monolithique du rôle des femmes dans la guerre à notre époque. L'archétype de la criminelle de guerre, qui nie l'idée d'une passivité propre au sexe féminin dans les conflits armés, participe à articuler les valeurs d'une société en ce qu'elle incarne, comme le soutient Lecercle, «cet Autre radical qui est essentiel à la construction du Même » (178). Cependant, la littérature va plus loin : elle remet ces valeurs en jeu, donc en cause. Les textes convoqués dans cet article ne font pas que « confort[er] par inversion » (178) les idées reçues sur la marginalité de l'usage de la violence chez les femmes, sur leur condition de victime et sur la solidarité féminine : ils s'appuient sur la familiarité du lecteur avec ces matériaux qui composent l'archétype de la femme criminelle pour faire émerger des propositions originales. À partir de récits qui ne différencient ni le sexe ni l'âge des bourreaux, et qui banalisent le phénomène de la perpétration de crimes de guerre par des individus que l'on n'associe pas traditionnellement à la violence et au crime, Courtemanche et Kourouma font état d'une indifférence généralisée envers des conflits dont la brutalité profane l'idée même d'humanité. Char et Mouawad suggèrent, quant 
à eux, qu'une histoire de violence sans issue pour les (anti)héroïnes qui la vivent peut, lorsque transmise, contribuer à combattre l'ignorance qui confine à la condition de victime et à l'esprit de vengeance. Les variations sur la figure de la criminelle de guerre que proposent ces récits contemporains complexifient enfin les archétypes qui forment les catégories usuelles - victime, protectrice, résistante - et font place à une plus grande diversité de figures, non pas pour singulariser celles-ci, mais pour redéfinir la norme.

\section{Ouvrages cités}

Amabiamina, Flora. «Visages et représentations de femmes dans les situations de conflit: l'exemple de Murambi le livre des ossements de Boris Boubacar Diop et d'Allah n'est pas obligé d’Ahmadou Kourouma ». LittéRéalité 19.2 (2007). 33-54.

Béchara, Souha. Résistante. Paris : Lattès, 2000.

Cardi, Coline et Geneviève Pruvost, dir. Penser la violence des femmes. Paris : La Découverte, 2017.

Char, Yasmine. La main de Dieu. Paris : Gallimard, 2008.

Chevillot, Frédérique et Colette Trout, dir. Rebelles et criminelles chez les écrivaines d'expression française. Amsterdam : Rodopi, 2013.

Courtemanche, Gil. Un dimanche à la piscine à Kigali. Montréal : Boréal, 2004.

Dayan-Herzbrun, Sonia. «Femmes du Liban et de la Palestine dans la lutte armée ». Cardi et Pruvost. 142-63.

Gentry, Caron E. et Laura Sjoberg. Beyond Mothers, Monsters, Whores : Thinking about Women's Violence in Global Politics. Londres : Zed, 2015.

Jouve, Emeline, Aurélie Guillain et Laurence Talairach-Vielmas, dir. L'acte inqualifiable, ou le meurtre au féminin / Unspeakable Acts : Murder by Women. Bruxelles : Lang, 2016.

Kravetz, Marc. «Profession : correspondant de guerre». Écrire la guerre. Dir. Laurent Nunez. Paris : Magazine littéraire, 2013. 43-53.

Kourouma, Ahmadou. Allah n'est pas obligé. Paris : Seuil, 2000.

Lecercle, Jean-Jacques. «La petite fille criminelle : archétype et littérature ». Jouve, Guillain et Talairach-Vielmas. 165-80.

Mouawad, Wajdi. Incendies. Montréal : Leméac, 2009 [2003]. 
Odaert, Olivier et François-Xavier Lavenne. « Les écrivains au cœur des discours de la guerre ». Interférences littéraires 3 (2009). 9-24.

Rabaté, Dominique. «L'abîme du banal. Réflexions sur le mal au XXe siècle ». Modernités 29 (2008) : 279-303.

---. «Logiques du pire : Un des malheurs et La scie patriotique ». Écritures de la guerre aux XXe et XXIe siècles. Dir. Marie-Hélène Boblet et Bernard Alazet. Dijon : PU de Dijon, 2010. 101-118.

\footnotetext{
Notes

${ }^{1}$ La part des femmes dans les horreurs commises au cours de la Deuxième Guerre mondiale est ainsi abordée par Daniel Patrick Brown dans The Camp Women : The Female Auxiliaries Who Assisted The SS in Running the Nazi Concentration Camp System (2002) et par Wendy Lower dans Hitler's Furies : German Women in the Nazi Killing Fields (2013). À propos de conflits plus récents, Slavenka Drakulić a consacré un chapitre de They Would Never Hurt a Fly : War Criminals on Trial in The Hague (2004) à Biljana Plavšić, ex-vice-présidente des Serbes de Bosnie, condamnée entre autres pour crimes contre l'humanité commis pendant la guerre de Yougoslavie, et Laura Sjoberg a fait paraître Women as Wartime Rapists : Beyond Sensation and Stereotyping (2017), couvrant différents contextes, de l'Allemagne nazie à ISIS. On peut également relever des témoignages, notamment ceux de Souha Béchara, jeune étudiante communiste ayant tenté d'assassiner Antoine Lahad, chef des milices du Sud pendant la guerre du Liban (2000 et 2014), ainsi qu'un projet artistique/commentaire critique par Coco Fusco intitulé Petit manuel de torture à l'usage des femmes-soldats (2008), motivé par des figures telles que Lynndie England, tristement célèbre pour avoir torturé des civils à Abou Ghraïb pendant la guerre étatsunienne en Irak.

${ }^{2}$ C'est là une des prémisses de l'ouvrage de Coline Cardi et Geneviève Pruvost. Caron E. Gentry et Laura Sjoberg affirment également d'entrée de jeu que la réalité de la violence politique employée par les femmes est davantage une « unseen long-term reality » (1) qu'une nouvelle tendance.
} 